\title{
Esculetin, a Coumarin Derivative, Exhibits Anti-proliferative and Pro-apoptotic Activity in G361 Human Malignant Melanoma
}

ORIGINAL

ARTICLE

\author{
Young-Joo Jeon ${ }^{1}$, Jeong-Yun Jang ${ }^{1}$, Jung-Hyun Shim ${ }^{2}$, Pyung Keun Myung ${ }^{3, *}$, Jung-II Chae ${ }^{1, *}$ \\ 'Department of Dental Pharmacology, School of Dentistry and Institute of Oral Bioscience, BK21 Plus, Chonbuk National University, Jeonju, \\ ${ }^{2}$ Department of Pharmacy, College of Pharmacy and Natural Medicine Research Institute, Mokpo National University, Muan, ${ }^{3}$ College of Pharmacy, \\ Chungnam National University, Daejeon, Korea
}

\begin{abstract}
Background: Although esculetin, a coumarin compound, is known to induce apoptosis in human cancer cells, the effects and molecular mechanisms on the apoptosis in human malignant melanoma (HMM) cells are not well understood yet. In this study, we investigated the anti-proliferative effects of esculetin on the G361 HMM cells.

Methods: We analyzed the anti-proliferative effects and molecular mechanisms of esculetin on G361 cells by a 3-(4,5-dimethylthiazol2-yl)-5-(3-carboxymethoxy phenyl)-2-(4-sulfophenyl)-2H-tetrazolium assay, 4',6-diamidino-2-phenylindole staining and Western blotting. Results: Esculetin exhibited significant anti-proliferative effects on the HMM cells in a dose-dependent manner. Interestingly, we found that esculetin induced nuclear shrinkage and fragmentation, typical apoptosis markers, by suppression of Sp1 transcription factor (Sp1). Notably, esculetin modulated Sp1 downstream target genes including p27, p21 and cyclin D1, resulted in activation of apoptosis signaling molecules such as caspase-3 and PARP in G361 HMM cells.

Conclusions: Our results clearly demonstrated that esculetin induced apoptosis in the HMM cells by downregulating Sp1 protein levels. Thus, we suggest that esculetin may be a potential anti-proliferative agent that induces apoptotic cell death in G361 HMM cells.

(J Cancer Prev 2015;20:106-112)
\end{abstract}

Key Words: Esculetin, Apoptosis, Melanoma, Sp1 transcription factor

\section{INTRODUCTION}

Esculetin, 6,7-dihydroxycoumarin, is a coumarin derivative found in various natural plant products such as Atremisia capillaries, Citrus limonia and Euphorbia lathyris. ${ }^{1}$ This compound possesses various biological and pharmaceutical properties including anti-edema, anti-inflammatory and antitumour effects, ${ }^{2-9}$ and as a result, it is also used to synthesize drugs. ${ }^{10}$ Esculetin was previously reported to be a scavenger of oxygen-free radicals that induce the apoptosis of human leukemia HL-60 cells and oral cancer SAS cells. ${ }^{11-14}$ However, the anti-proli- ferative activity of esculetin in human malignant melanoma (HMM) cells and its underlying molecular mechanisms are not yet completely understood.

HMM is the deadliest form of skin cancer and is responsible for $75.2 \%$ of deaths related to skin cancer. ${ }^{15}$ Furthermore, the incidence of a melanoma has continued to increase in the white population worldwide, and the most notable increases have been observed in in situ melanomas. ${ }^{16,17}$ The death rate varies by gender, country, and ethnic origin, and there were an estimated 53,480 new cases and 8,420 (13.5\%) deaths in the United States in 2008. ${ }^{15}$ Patients with an early-stage diagnosis can be cured

Received May 27, 2015, Revised June 9, 2015, Accepted June 9, 2015

Correspondence to: Pyung Keun Myung

College of Pharmacy, Chungnam National University, 99 Daehak-ro, Yuseong-gu, Daejeon 305-764, Korea

Tel: +82-42-821-5929, Fax: +82-42-823-6566, E-mail: pyung@cnu.ac.kr, ORCID: Pyung Keun Myung, http://orcid.org/0000-0001-9980-8568

Correspondence to: Jung-Il Chae

Department of Oral Pharmacology, School of Dentistry, BK21 Plus, Chonbuk National University, 567 Baekje-daero, Deokjin-gu, Jeonju 561-756, Korea

Tel: +82-63-270-4024, Fax: +82-63-270-4037, E-mail: jichae@jbnu.ac.kr, ORCID: Jung-Il Chae, http://orcid.org/0000-0003-2232-077X

*These authors contributed equally to this paper as co-correspondencing authors.

Copyright (C) 2015 Korean Society of Cancer Prevention

(c) This is an Open Access article distributed under the terms of the Creative Commons Attribution Non-Commercial License (http://creativecommons.org/licenses/by-nc/4.0) which permits unrestricted non-commercial use, distribution, and reproduction in any medium, provided the original work is properly cited. 
through a surgical excision while the prognosis of patients with distant metastases that are not amenable to surgery remains poor. ${ }^{18}$ The median survival rate reported in clinical trials for most patients with stage IV melanoma is an average of only 7 to 9 months. ${ }^{19}$ A unique hallmark of HMM is that it is highly resistant to apoptosis, and the mechanisms by which melanoma cells protect themselves from drug-induced apoptosis remain largely unknown. ${ }^{20}$ Therefore, an understanding of the apoptosis of HMM cells is a prerequisite for further breakthroughs in the therapy for HMM patients.

The Sp1 transcription factor (Sp1) is a member of the zinc-finger Sp family of proteins that includes the Kruppel-like factor family with members that share a highly conserved DNA-binding domain (sequence identity more than 65\%) with three adjacent Cys2His2-type zinc fingers that bind to GC- and/or GT-boxes with overlapping specificities and affinities. ${ }^{21-24}$ Furthermore, Sp1 increases greatly in various types of cancers, and this condition could be a critical factor for tumour development or maintenance. ${ }^{25}$ As a result, many studies have suggested that Sp1-mediated functions are novel targets for cancer therapy.

Although esculetin has been clearly demonstrated to have a biological activity against a number of cancer cells, its effects on HMM and the mechanisms behind esculetin-induced apoptosis are not yet understood. Therefore, this study investigates the pro-apoptotic activity of esculetin as well as the molecular mechanisms for such activity in HMM cell lines.

\section{MATERIALS AND METHODS}

\section{Cell culture}

G361 (CRL-1424) is a HMM cell line that can be obtained from American Type Culture Collection (ATCC, Rockville, MD, USA). The cells were maintained in Dulbecco's modified Eagle's medium (Welgene, Daegu, Korea) supplemented with 10\% fetal bovine serum and $100 \mathrm{U} / \mathrm{mL}$ each of penicillin and streptomycin (Gibco, Grand Island, NY, USA) with appropriate concentrations at $37^{\circ} \mathrm{C}$ with $5 \% \mathrm{CO}_{2}$ in a fully humidified atmosphere.

\section{Cell viability assay}

The effect of esculetin on cell viability was estimated using a MTS assay kit (Promega, Madison, WI, USA). The G361 cells were seeded overnight at a density of $3 \times 10^{3}$ cells per well in 96-well plates in complete growth medium and were then treated with the relevant esculetin for 24 or 48 hours. The absorbance was measured using an Absorbance Microplate Reader (Biotek,
Winooski, VT, USA) at $490 \mathrm{~nm}$, and all experiments were carried out in triplicate and the percentages for the cell viability of esculetin-treated cells were normalized to that of untreated control cells.

\section{4',6-diamidino-2-phenylindole staining}

The nuclear condensation and fragmentation level was observed via nucleic acid staining with 4',6'-diamidine-2'phenylindole (DAPI) dihydrochloride. The G361 cells were treated with various concentrations of esculetin, were harvested via trypsinization, washed with ice-cold phosphate buffered saline (PBS) and then fixed in $100 \%$ methanol at room temperature for 20 minutes. The cells were spread on a slide and were stained with a DAPI (Sigma-Aldrich, St. Louis, MO, USA) solution $(2 \mu \mathrm{g} / \mathrm{mL}$ ). Fluorescence images were then taken using a FluoView confocal laser microscope (Fluoview FV10i; Olympus, Tokyo, Japan).

\section{Western blot analysis}

The protein expression levels were analyzed via Western blotting. After the G361 cells were treated with esculetin, the cells were washed with ice-cold PBS and were then lysed in an ice-cold M-PER ${ }^{\circledR}$ Mammalian Protein Extraction Reagent (Thermo Scientific, Rockford, IL, USA) containing a protease inhibitor cocktail (Roche, Basel, Switzerland). The soluble fraction of the cell lysates was isolated by centrifugation at $13,000 \times g$ for 15 minutes in a microfuge. A BCA Protein Assay Kit (Thermo Scientific) was used to measure the protein concentration in the supernatants, and equal amounts of protein samples were separated by $10 \%$ or $12 \%$ SDS-polyacrylamide gel electrophoresis and were then transferred to polyvinylidene difluoride membranes (Millipore, Billerica, MA, USA), which were blocked for 30 minutes at room temperature with $5 \%(\mathrm{v} / \mathrm{v})$ skim milk in TBS buffer containing $0.1 \%$ tween-20. The samples were then incubated with primary antibodies overnight at $4^{\circ} \mathrm{C}$. The primary antibodies used in this study were as followings: $\alpha$-Sp1, $\alpha$-p27, $\alpha$-p21, $\alpha$-cyclinD1 (Santa Cruz Biotechnology Inc., Santa Cruz, CA, USA), $\alpha$-procaspase 3, $\alpha$-cleaved caspase 3 (Cell Signaling Technology, Denvers, MA, USA), and glyceraldehyde-3-phosphate dehydrogenase (GAPDH) (AbFrontier, Seoul, Korea). The membranes were washed for 10 minutes 5 times with TBS-T and were incubated with horseradish-peroxidase-conjugated anti-mouse immunoglobulin G (IgG) or anti-rabbit IgG (Santa Cruz Biotechnology Inc.). The membranes were then washed 5 times for 10 minutes with TBS-T and the chemiluminescence signals were enhanced using a Pierce ECL Western Blotting Substrate (Thermo Scientific) and detected using ImageQuant Las4000 Mini (GE 
A<smiles>O=c1ccc2cc(O)c(O)cc2o1</smiles>

Esculetin
B

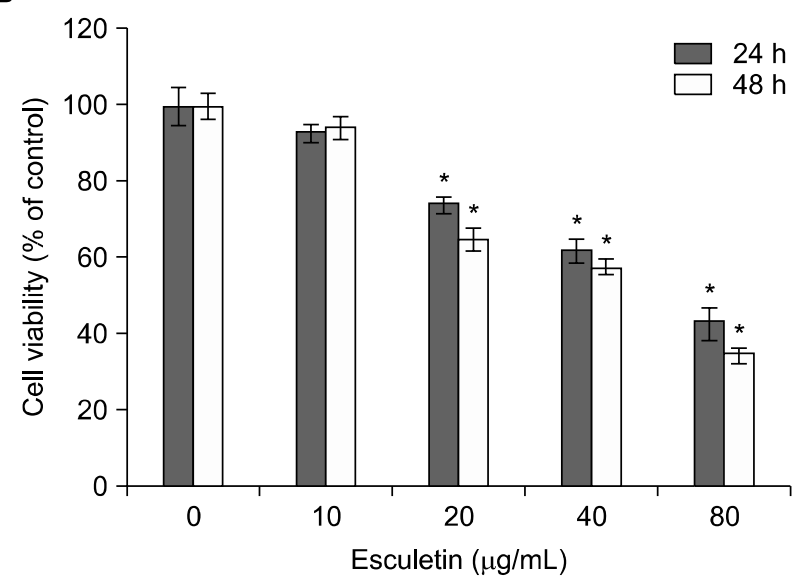

C

Esculetin $(\mu \mathrm{g} / \mathrm{mL})$
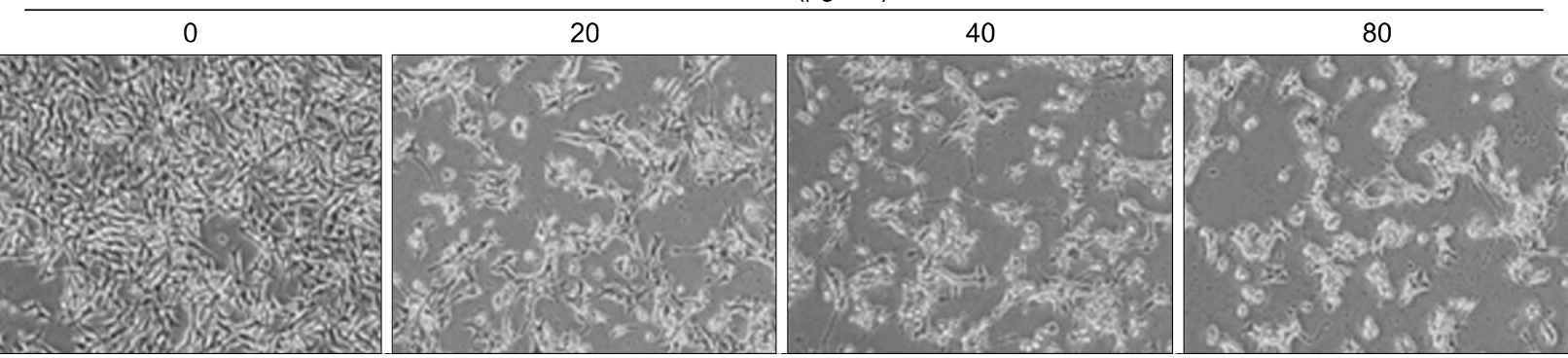

Figure 1. The effect of esculetin on the cell viability in G361 cells. (A) Chemical structure of esculetin. (B) Cell viability effects of esculetin on G361 cells. The G361 cells were treated with esculetin $(0,10,20,40$, and $80 \mu \mathrm{g} / \mathrm{mL})$ for 24 hours and 48 hours, and the cell viabilities were measured with an MTS assay. The asterisk indicates a significant difference relative to untreated control cells $(* P<0.05)$. (C) Changes in the cell morphologies in G361 cells treated or untreated with esculetin $(0,20,40$, and $80 \mu \mathrm{g} / \mathrm{mL})$ for 48 hours $(\times 40)$.

Healthcare Life Sciences, Little Chalfont, UK).

\section{Statistical analysis}

The results are presented as means \pm standard deviation of triplicate independent experiments. A Student's $t$-test was conducted, and the statistical significance was considered assessed for a $P$-value less than 0.05 relative to untreated control (SigmaPlot ver. 10.0; Systat Software Inc., San Jose, CA, USA).

\section{RESULTS}

\section{Esculetin inhibits cell viability of a human malignant melanoma cell line}

To examine the effects of esculetin (Fig. 1A) on the cell proliferation in G361 cells, we conducted MTS assay to evaluate the viability. We found that the cell viability of G361 cells was decreased at both 24 hours and 48 hours by treatment of esculetin in a dose dependent manner (Fig. 1B). When G361 HMM cells were treated with esculetin for 48 hours, the cell viability was $94 \%, 65 \%, 57 \%$, and $34 \%$ at $10,20,40$, and $80 \mu \mathrm{g} / \mathrm{mL}$ compared with a vehicle treated control group, respectively (Fig. 1B). The $\mathrm{IC}_{50}$ value on the cell viability was about $42.86 \mu \mathrm{g} / \mathrm{mL}$ in G361 HMM cells at 48 hours by esculetin treatment. We further observed that esculetin induced severe morphological changes including cytoplasmic blebbing, irregular cell morphology change and detachment from cell culture vessels in a dose dependent manner (Fig. 1C). These results strongly suggest that esculetin inhibits G361 HMM cells.

\section{Esculetin-induced apoptosis of human malignant melanoma cell line}

The morphological changes including cytoplasmic blebbing, irregular cell morphology change and detachment from cell culture vessels are critical hallmarkers of apoptosis. ${ }^{26}$ To determine whether esculetin induced apoptosis or not, we examined the nuclei integrity by esculetin treatment. G361 HMM cells were treated with $60 \mu \mathrm{g} / \mathrm{mL}$ of esculetin for 48 hours and stained with DAPI. We found that esculatin dramatically induced nuclei condensation and fragmentation compared with dimethyl sulfoxide treated control cells (Fig. 2). These results demonstrated that 
A
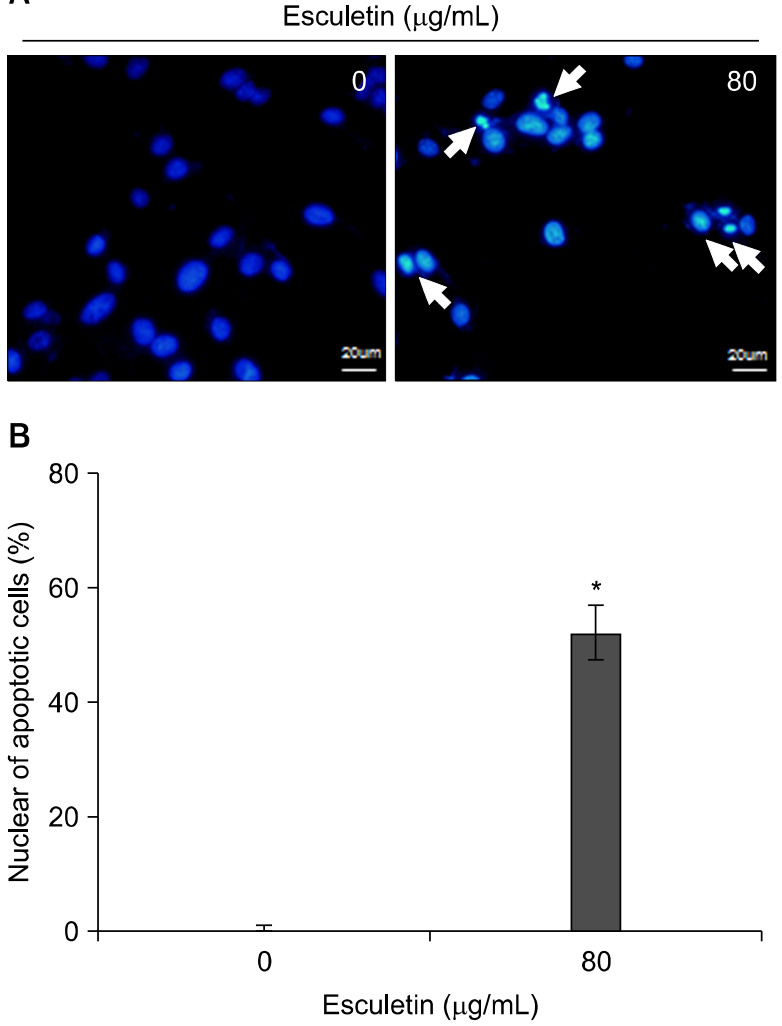

Figure 2. Apoptotic effect induced by esculetin in human malignant melanoma cells. The G361 cells were incubated with esculetin ( 0 and $80 \mu \mathrm{g} / \mathrm{mL}$ ) for 48 hours. (A) Fluorescence microscopy images of the DAPI-stained cells. The white arrows represent DNA fragmentation and chromatin condensation. Scale bars: $20 \mu \mathrm{m}$. (B) The DNA fragmentation and chromatin condensation were determined to have a significant difference relative to untreated control cells, as indicated by an asterisk $(* P<0.05)$.

esculetin suppressed cell proliferation by induction of apoptosis.

\section{Esculetin suppresses Sp1 expression in a human malignant melanoma cell line}

Previous studies demonstrated that activity and protein levels of the transcription factor Sp1 were well correlated with the human cancer development. ${ }^{27}$ To examine whether esculetin protein levels were involved in esculetin-mediated cell proliferation and apoptosis, G361 cells were treated with esculetin for 48 hours as indicated. We found that esculetin suppressed Sp1 protein levels in a dose dependent manner (Fig. 3A). Notably, we observed that $80 \mu \mathrm{g} / \mathrm{mL}$ of esculetin suppressed Sp1 protein levels and induced caspase- 3 cleavage in a time dependent manner (Fig. 3B). Therefore, the down-regulation of Sp1 by treatment with esculetin leads to apoptotic cell death.

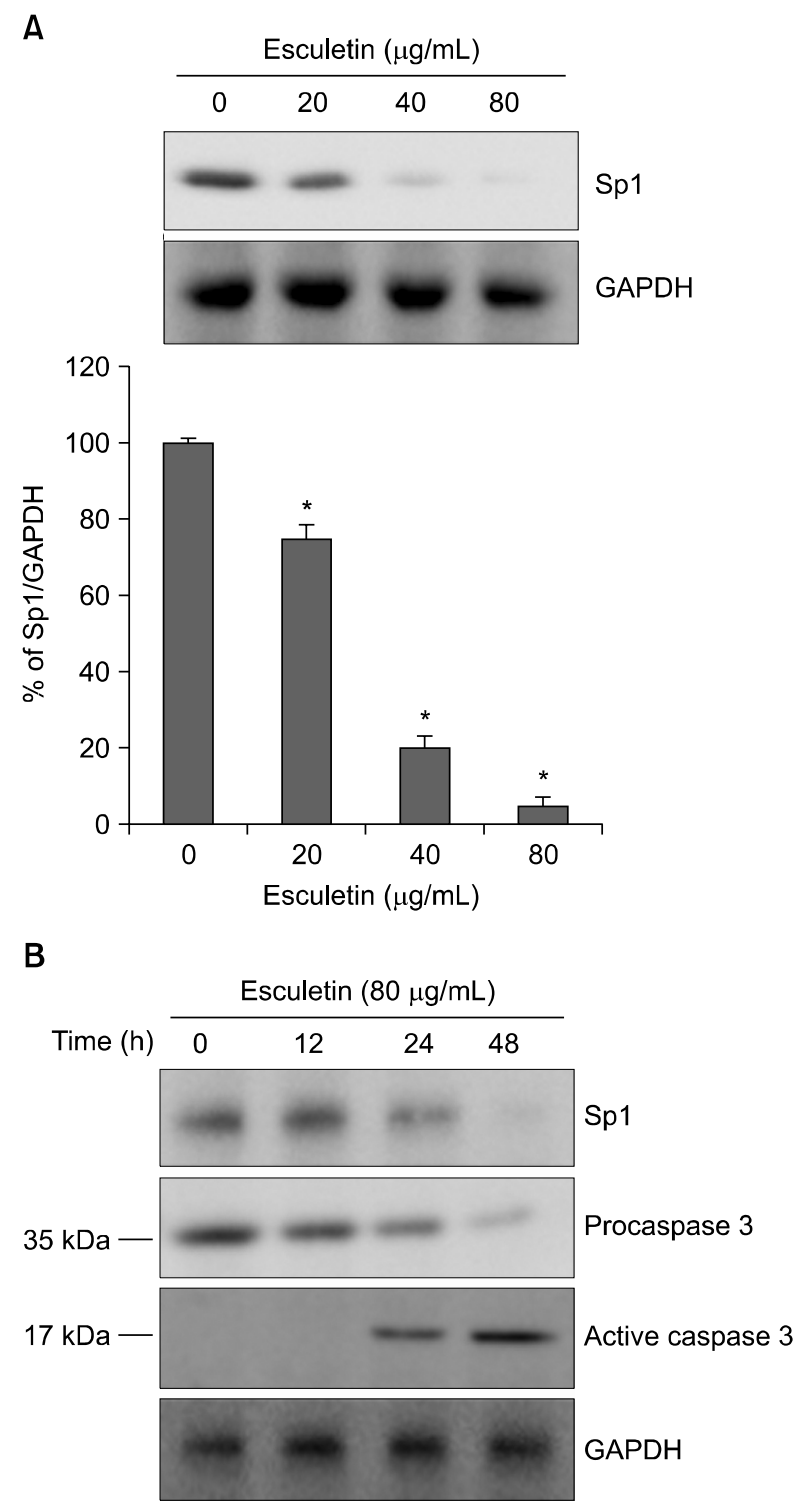

Figure 3. The effect of esculetin on Sp1 expression in human malignant melanoma. (A) The G361 cells were treated with different concentrations of esculetin $(0,20,40$, and $80 \mu \mathrm{g} / \mathrm{mL})$ for 48 hours. The cell lysates were separated by SDS PAGE, and then the membranes were transferred from SDS PAGE gels subjected to Western blot analysis for Sp1. An equal loading protein was confirmed using glyceraldehyde-3-phosphate dehydrogenase (GAPDH). The histogram showed the ratio of Sp1 to GAPDH expression, and the results were expressed as the average of a triplicate sample from three independent experiments. The asterisk suggested $P<0.05$ versus untreated control cells. (B) The G361 cells were treated with $80 \mu \mathrm{g} / \mathrm{mL}$ of esculetin for $0,12,24$, and 48 hours, and then the cells were evaluated in terms of the expression level of Sp1, procaspase 3 and active caspase 3. Equal loading protein was confirmed using GAPDH. 
A

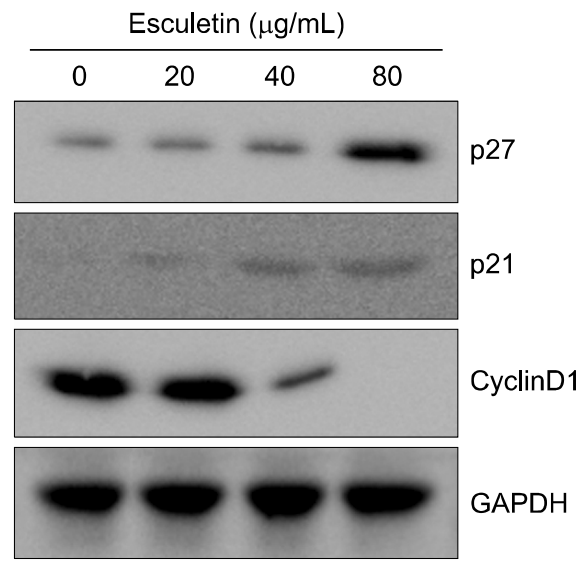

B

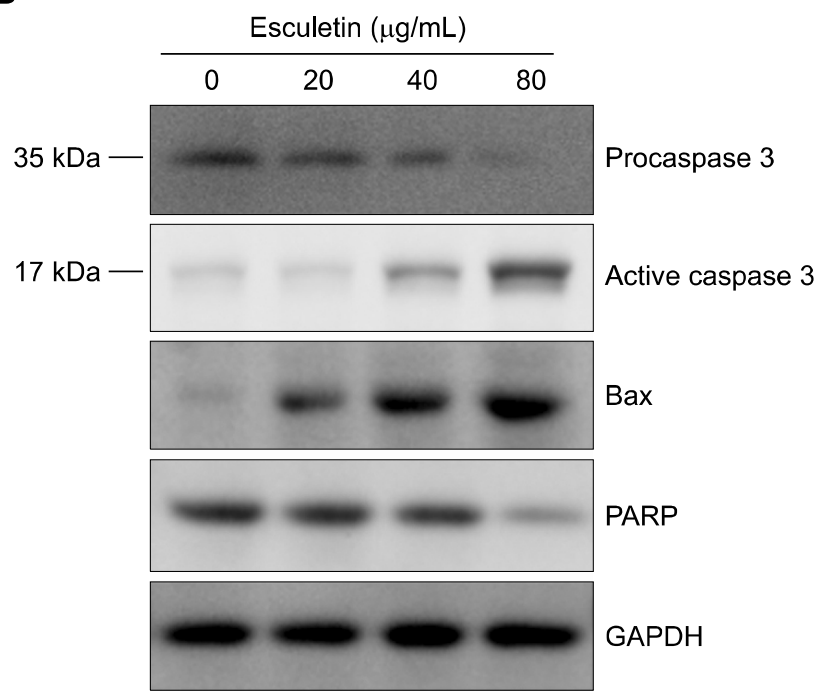

Figure 4. The effect of esculetin on the expression of cell cycle arrest- and apoptosis-related proteins in human malignant melanoma. (A) The G361 cells were incubated with esculetin $(20,40$, and $80 \mu \mathrm{g} / \mathrm{mL})$ or without for 48 hours. The cell lysates were determined using Western blot analysis with antibodies against p27, p21, and cyclin D1. Equal loading protein was confirmed using glyceraldehyde-3-phosphate dehydrogenase (GAPDH). (B) The G361 cells were treated with esculetin $(0,20,40$, and $80 \mu \mathrm{g} / \mathrm{mL})$ for 48 hours. The cell lysates were then determined via Western blot analysis with antibodies against procaspase 3, active caspase 3. Bax, and PARP. Equal loading protein was confirmed using GAPDH. The values were measured using Image J densitometry, and the data were expressed as a representative of two or three independent experiments.

\section{Esculetin regulates the expression of cell cycle} arrest- and apoptosis-related proteins in a human malignant melanoma cell line

The treatment of cells with esculetin regulated the expression levels of several cell cycle related proteins (p27, p21, and cyclin D1) (Fig. 4A) and apoptosis related proteins (procaspase 3, active caspase 3, Bax, and PARP) (Fig. 4B). The G361 cells were treated with different doses of esculetin for 48 hours and were harvested. The protein expression levels of p27, p21, cyclin D1, procaspase 3, active caspase 3, Bax, and PARP were analyzed by Western blotting. The results indicate that esculetin increased p21, p27, Bax and active caspase 3 protein levels and decreased cyclinD1, procaspase 3 , and PARP protein levels in the G361 cells. These results suggest that esculetin treatment in G361 cells suppresses Sp1 protein levels, resulting in cell cycle arrest and apoptotic cell death.

\section{DISCUSSION}

Esculetin is a naturally-occurring coumarin derivative that shows chemopreventive and chemotherapeutic activity against several types of cancers. ${ }^{28}$ This compound can play an important role as a potential agent against anti-proliferation. In this regard, several studies have reported that esculetin has an anti- proliferative effect on various cancer derived cells, including smooth vascular muscle cells and human leukemia cells. ${ }^{5.14}$ However, the mechanisms underlying its anti-proliferative effect on HMM have not yet been determined. In this study we have clarified the molecular mechanisms through which esculetin triggers apoptosis in HMM G361 cells.

HMM is a cancer of the skin, and its incidence has increased over the last 50 years in most of the fair-skinned population. ${ }^{29}$ Despite the availability of several treatment options, including surgery, radiation, chemotherapy or combinations thereof, HMM still has low survival rates. Thus, clinically effective agents are highly required for HMM treatments. The esculetin treatment of the G361 cells with different times and concentrations resulted in a decrease in cell size, and the cells became rounded during apoptosis (Fig. 1 and 2).

The Sp1 transcription factor is highly overexpressed in several human cancer cell lines and plays an important role in a variety of cellular processes through the regulation of its target genes. ${ }^{30,31}$ Therefore, numerous studies have investigated whether Spl that is downregulated by anti-proliferative agents modulates cell cycle-related proteins or apoptosis-related proteins, which lead to an inhibition of the proliferation and apoptosis. ${ }^{32.33}$ Thus, Sp1 could be an ideal molecular target for molecular therapy against cancer.

In this study, Sp1 expression significantly decreased in the 
esculetin-treated G361 cells (Fig. 3). To determine whether esculetin can change the target protein expression of Sp1 and apoptosis-related proteins in HMM, we investigated the expression of these proteins in close relation with cell cycle arrest and survival. The results provide insight into the mechanism for the anti-proliferative effect in HMM. The results indicate that esculetin also regulated the Sp1 target proteins, such as $\mathrm{p} 27, \mathrm{p} 21$, and cyclinD1 (Fig. 4A). The p27 and p21 proteins act primarily by inhibiting the kinase activity of cyclin-dependent kinase, resulting in a blocked cell cycle progression. ${ }^{34,35}$ The cyclin D1 is indispensable for cell cycle progression and is related to cell maintenance and tumorigenesis due to its promotion of the G1/S phase transition. ${ }^{36}$ In addition, esculetin induced Bax and also activated caspase-3 and PARP (Fig. 4B), suggesting that esculetin regulated Sp1 and finally led to an apoptotic cell death.

In this study, we have investigated the cancer chemoprevention effect of esculetin on HMM. Esculetin appears to inhibit cell proliferation and to induce apoptosis by Sp1 through Sp1 target proteins and apoptosis-related genes. Altogether, esculetin might be a promising therapeutic agent for HMM treatment.

\section{ACKNOWLEDGMEMTS}

This work was supported by the Agenda Program (PJ00932102) from the Rural Development Administration, Republic of Korea and Research Fund from Chungnam National University.

\section{CONFLICTS OF INTEREST}

No potential conflicts of interest were disclosed.

\section{REFERENCES}

1. Masamoto Y, Ando H, Murata Y, Shimoishi Y, Tada M, Takahata K. Mushroom tyrosinase inhibitory activity of esculetin isolated from seeds of Euphorbia lathyris L. Biosci Biotechnol Biochem 2003;67:631-4.

2. Payá M, Halliwell B, Hoult JR. Interactions of a series of coumarins with reactive oxygen species. Scavenging of superoxide, hypochlorous acid and hydroxyl radicals. Biochem Pharmacol 1992;44:205-14

3. Yang J, Xiao YL, He XR, Qiu GF, Hu XM. Aesculetin-induced apoptosis through a ROS-mediated mitochondrial dysfunction pathway in human cervical cancer cells. J Asian Nat Prod Res 2010; 12:185-93.

4. Huang HC, Lai MW, Wang HR, Chung YL, Hsieh LM, Chen CC. Antiproliferative effect of esculetin on vascular smooth muscle cells: possible roles of signal transduction pathways. Eur J Pharmacol 1993;237:39-44.

5. Matsunaga K, Yoshimi N, Yamada Y, Shimizu M, Kawabata K,
Ozawa $\mathrm{Y}$, et al. Inhibitory effects of nabumetone, a cyclooxygenase-2 inhibitor, and esculetin, a lipoxygenase inhibitor, on $\mathrm{N}$-methyl-N-nitrosourea-induced mammary carcinogenesis in rats. Jpn J Cancer Res 1998;89:496-501.

6. Hecht SS, Kenney PM, Wang M, Trushin N, Agarwal S, Rao AV, et al. Evaluation of butylated hydroxyanisole, myo-inositol, curcumin, esculetin, resveratrol and lycopene as inhibitors of benzo[a]pyrene plus 4-(methylnitrosamino)-1-(3-pyridyl)-1-butanoneinduced lung tumorigenesis in A/J mice. Cancer Lett 1999;137: 123-30.

7. Kim $\mathrm{AD}$, Han X, Piao MJ, Hewage SR, Hyun CL, Cho SJ, et al. Esculetin induces death of human colon cancer cells via the reactive oxygen species-mediated mitochondrial apoptosis pathway. Environ Toxicol Pharmacol 2015;39:982-9.

8. Kimura Y, Sumiyoshi M. Antitumor and antimetastatic actions of dihydroxycoumarins (esculetin or fraxetin) through the inhibition of M2 macrophage differentiation in tumor-associated macrophages and/or G1 arrest in tumor cells. Eur J Pharmacol 2015;746:115-25.

9. Cho JH, Shin JC, Cho JJ, Choi YH, Shim JH, Chae JI. Esculetin (6,7-dihydroxycoumarin): a potential cancer chemopreventive agent through suppression of Sp1 in oral squamous cancer cells. Int J Oncol 2015:46:265-71.

10. Kostova I. Synthetic and natural coumarins as cytotoxic agents. Curr Med Chem Anticancer Agents 2005;5:29-46.

11. Egan D, O'Kennedy R, Moran E, Cox D, Prosser E, Thornes RD. The pharmacology, metabolism, analysis, and applications of coumarin and coumarin-related compounds. Drug Metab Rev 1990; 22:503-29.

12. Okada Y, Miyauchi N, Suzuki K, Kobayashi T, Tsutsui C, Mayuzumi K, et al. Search for naturally occurring substances to prevent the complications of diabetes. II. Inhibitory effect of coumarin and flavonoid derivatives on bovine lens aldose reductase and rabbit platelet aggregation. Chem Pharm Bull (Tokyo) 1995:43:1385-7.

13. Kok SH, Yeh CC, Chen ML, Kuo MY. Esculetin enhances TRAIL-induced apoptosis through DR5 upregulation in human oral cancer SAS cells. Oral Oncol 2009;45:1067-72.

14. Wang CJ, Hsieh YJ, Chu CY, Lin YL, Tseng TH. Inhibition of cell cycle progression in human leukemia HL-60 cells by esculetin. Cancer Lett 2002;183:163-8.

15. Su DM, Zhang Q, Wang X, He P, Zhu YJ, Zhao J, et al. Two types of human malignant melanoma cell lines revealed by expression patterns of mitochondrial and survival-apoptosis genes: implications for malignant melanoma therapy. Mol Cancer Ther 2009:8:1292-304.

16. Gray-Schopfer V, Wellbrock C, Marais R. Melanoma biology and new targeted therapy. Nature 2007;445:851-7.

17. MacKie RM, Bray C, Vestey J, Doherty V, Evans A, Thomson D, et al; Scottish Melanoma Group. Melanoma incidence and mortality in Scotland 1979-2003. Br J Cancer 2007;96:1772-7.

18. Bhatia S, Tykodi SS, Thompson JA. Treatment of metastatic melanoma: an overview. Oncology (Williston Park) 2009;23:488-96.

19. Middleton MR, Grob JJ, Aaronson N, Fierlbeck G, Tilgen W, Seiter $\mathrm{S}$, et al. Randomized phase III study of temozolomide versus dacarbazine in the treatment of patients with advanced metastatic malignant melanoma. J Clin Oncol 2000;18:158-66.

20. Meier F, Satyamoorthy K, Nesbit M, Hsu MY, Schittek B, Garbe C, et al. Molecular events in melanoma development and progression. 
Front Biosci 1998:3:D1005-10.

21. Black AR, Black JD, Azizkhan-Clifford J. Sp1 and krüppel-like factor family of transcription factors in cell growth regulation and cancer. J Cell Physiol 2001;188:143-60.

22. Cook T, Gebelein B, Urrutia R. Sp1 and its likes: biochemical and functional predictions for a growing family of zinc finger transcription factors. Ann N Y Acad Sci 1999;880:94-102.

23. Kaczynski J, Cook T, Urrutia R. Sp1- and Krüppel-like transcription factors. Genome Biol 2003:4:206.

24. Lomberk G, Urrutia R. The family feud: turning off $\mathrm{Sp} 1$ by Sp1-like KLF proteins. Biochem J 2005:392:1-11.

25. Davie JR, He S, Li L, Sekhavat A, Espino P, Drobic B, et al. Nuclear organization and chromatin dynamics: Sp1, Sp3 and histone deacetylases. Adv Enzyme Regul 2008;48:189-208.

26. Saraste A, Pulkki K. Morphologic and biochemical hallmarks of apoptosis. Cardiovasc Res 2000;45:528-37.

27. Sankpal UT, Goodison S, Abdelrahim M, Basha R. Targeting Sp1 transcription factors in prostate cancer therapy. Med Chem 2011;7:518-25.

28. Chang WS, Lin CC, Chuang SC, Chiang HC. Superoxide anion scavenging effect of coumarins. Am J Chin Med 1996:24:11-7.

29. Ferlay J, Steliarova-Foucher E, Lortet-Tieulent J, Rosso S, Coebergh JW, Comber H, et al. Cancer incidence and mortality patterns in Europe: estimates for 40 countries in 2012. Eur J Cancer
2013:49:1374-403.

30. Deng J, Qian Y, Geng L, Chen J, Wang X, Xie H, et al. Involvement of p38 mitogen-activated protein kinase pathway in honokiol-induced apoptosis in a human hepatoma cell line (hepG2). Liver Int 2008:28:1458-64.

31. Knappskog S, Lønning PE. Effects of the MDM2 promoter SNP285 and SNP309 on Sp1 transcription factor binding and cancer risk. Transcription 2011:2:207-10.

32. Kavurma MM, Khachigian LM. Sp1 inhibits proliferation and induces apoptosis in vascular smooth muscle cells by repressing p21WAF1/Cip1 transcription and cyclin D1-Cdk4-p21WAF1/Cip1 complex formation. J Biol Chem 2003;278:32537-43.

33. Xu R, Zhang P, Huang J, Ge S, Lu J, Qian G. Sp1 and Sp3 regulate basal transcription of the survivin gene. Biochem Biophys Res Commun 2007;356:286-92.

34. Yang ZY, Perkins ND, Ohno T, Nabel EG, Nabel GJ. The p21 cyclin-dependent kinase inhibitor suppresses tumorigenicity in vivo. Nat Med 1995;1:1052-6.

35. Wang X, Gorospe M, Huang Y, Holbrook NJ. p27Kip1 overexpression causes apoptotic death of mammalian cells. Oncogene 1997:15:2991-7.

36. Ewen ME, Lamb J. The activities of cyclin D1 that drive tumorigenesis. Trends Mol Med 2004;10:158-62. 